\title{
EULAR recommendations for the health professional's approach to pain management in inflammatory arthritis and osteoarthritis
}

\author{
Rinie Geenen, ${ }^{1}$ Cécile L Overman, ${ }^{1}$ Robin Christensen, ${ }^{2,3}$ Pernilla Åsenlöf, \\ Susana Capela, ${ }^{5,6}$ Karen L Huisinga, ${ }^{7}$ Mai Elin P Husebo,${ }^{8}$ Albère J A Köke, ${ }^{9}$ \\ Zoe Paskins, ${ }^{10,11}$ Irene A Pitsillidou, ${ }^{12}$ Carine Savel, ${ }^{13}$ Judith Austin, ${ }^{1}$ Afton L Hassett, ${ }^{14}$ \\ Guy Severijns, ${ }^{15}$ Michaela Stoffer-Marx, ${ }^{16,17}$ Johan W S Vlaeyen, ${ }^{18,19}$ \\ César Fernández-de-las-Peñas, ${ }^{20}$ Sarah J Ryan, ${ }^{11}$ Stefan Bergman ${ }^{21}$
}

\begin{abstract}
Handling editor Josef S Smolen

- Additional material is published online only. To view please visit the journal online (http://dx.doi.org/10.1136/ annrheumdis-2017-212662).
\end{abstract}

For numbered affiliations see end of article.

Correspondence to Professor Rinie Geenen, Department of Psychology, Utrecht University, Heidelberglaan 1, 3584CS Utrecht, The Netherlands; r.geenen@uu.nl

Received 7 November 2017 Revised 21 March 2018 Accepted 28 March 2018
Check for updates

To cite: Geenen $\mathrm{R}$, Overman $\mathrm{CL}$, Christensen $\mathrm{R}$, et al. Ann Rheum Dis 2018:77:797-807.

\section{ABSTRACT}

Pain is the predominant symptom for people with inflammatory arthritis (IA) and osteoarthritis (OA) mandating the development of evidence-based recommendations for the health professional's approach to pain management. A multidisciplinary task force including professionals and patient representatives conducted a systematic literature review of systematic reviews to evaluate evidence regarding effects on pain of multiple treatment modalities. Overarching principles and recommendations regarding assessment and pain treatment were specified on the basis of reviewed evidence and expert opinion. From 2914 review studies initially identified, 186 met inclusion criteria. The task force emphasised the importance for the health professional to adopt a patient-centred framework within a biopsychosocial perspective, to have sufficient knowledge of IA and OA pathogenesis, and to be able to differentiate localised and generalised pain. Treatment is guided by scientific evidence and the assessment of patient needs, preferences and priorities; pain characteristics; previous and ongoing pain treatments; inflammation and joint damage; and psychological and other pain-related factors. Pain treatment options typically include education complemented by physical activity and exercise, orthotics, psychological and social interventions, sleep hygiene education, weight management, pharmacological and joint-specific treatment options, or interdisciplinary pain management. Effects on pain were most uniformly positive for physical activity and exercise interventions, and for psychological interventions. Effects on pain for educational interventions, orthotics, weight management and multidisciplinary treatment were shown for particular disease groups. Underpinned by available systematic reviews and meta-analyses, these recommendations enable health professionals to provide knowledgeable pain-management support for people with IA and OA.

\section{INTRODUCTION}

Pain is the predominant symptom in the majority of people with inflammatory arthritis (IA) and osteoarthritis (OA) which both broadly contribute to the global burden of rheumatic and musculoskeletal conditions. $^{1-5}$ Knowledgeable pain-management support can reduce pain, increase functioning and well-being, and reduce individual and societal costs. ${ }^{6}$
Therefore, practitioners in all healthcare settings should have the knowledge and skills required to help people with IA and OA to better manage their pain. Rheumatology health professionals are ideally placed to provide comprehensive, evidence-based and patient-centred care.

Pain is a complex and multifaceted experience. Besides pathological processes such as inflammation and tissue damage, multiple individual factors influence pain, for example, illness beliefs, mood, avoidance behaviour, obesity, sleep disturbance, and the pattern of rest and activity throughout the day. ${ }^{8-13}$ These factors are commonly mentioned in educational materials and are part of the pain-management approach by health professionals in rheumatology. ${ }^{914} 15$

Meta-analyses and randomised controlled trials (RCTs) have, for instance, been performed with respect to pharmacological pain treatment in OA, ${ }^{1617}$ aerobic exercise in rheumatoid arthritis (RA) and $\mathrm{OA},{ }^{18}{ }^{19}$ activity pacing in chronic pain, ${ }^{20}$ and broad education and self-management approaches in rheumatic diseases. ${ }^{15} 21$ Evidence regarding pain-management support in IA and OA ranges from RCTs to expert reports, but as yet the effect of pain-management options on pain have not been studied in a comprehensive way for multiple pain treatment modalities. The aim of this review was to evaluate the existing scientific evidence associated with the benefits of the health professional's approach to pain management for people with IA and OA, and to use this evidence and expert opinion to provide recommendations that enable health professionals to provide knowledgeable pain-management support.

\section{METHODS}

The standardised operating procedures for European League Against Rheumatism (EULAR)-endorsed recommendations were followed, ${ }^{22}$ including a systematic literature review and definition of the strength of recommendations by a task force of experts. In the current article, the recommendations regarding management options mostly include advice with sufficient data-driven evidence, whereas the overarching principles and recommendations regarding assessment are based on expert opinion because they could not be substantiated with evidence from systematic reviews. 


\section{Task force}

The task force that included 18 members (16 from Europe and 2 from the USA) from 12 countries consisted of patient representatives, nurses, physiotherapists, psychologists, rheumatologists, a general practitioner, an occupational therapist, a clinical epidemiologist and a research fellow. The executive committee of the task force consisted of a convener (RG), methodologist (RC) and research fellow (CLO). During the first of two task force meetings, the research questions, scope of the project and pain-management options were defined.

\section{Scope}

Definition of the scope and framing of questions addressing management options in systematic reviews helped the task force to achieve focused recommendations. This process was guided by PICO which specifies the patient Population, Interventions, Comparator and Outcomes of interest. ${ }^{23}$

The target users of the recommendations are health professionals in the field of rheumatology including rheumatologists. The target population for these recommendations are patients with OA and patients with the following types of IA: RA, spondyloarthritis ( $\mathrm{SpA}$ ) and psoriatic arthritis (PsA).

It was decided that recommendations should cover core general pain management that virtually any health professional should be able to give including the appraisal of treatment options which require referral to specialised pain treatment. These options requiring referral included in the recommendations should be readily available to most healthcare practitioners. Moreover, it was decided to exclude general pharmacological and joint-specific medical and surgical treatments such as arthroplasty and glucocorticoid injections from the systematic literature review because these are better covered by the existing EULAR task force recommendations for the management of IA and OA. ${ }^{16} 17$ 24-29

To restrict the systematic literature review to pain-management strategies, the target outcome of our systematic review was pain. However, consistent with other expert groups, ${ }^{30} 31$ the task force unanimously endorsed that, besides pain, physical functioning (eg, activity) and psychological functioning (eg, emotional well-being and participation) are core domains of any management intervention in rheumatic care. This focus on pain as an outcome but also the multiple management options that are reviewed and the broad group of patients to which this study is relevant, differentiates our study from studies with a more general focus on education in IA, ${ }^{21}$ or non-pharmacological management of OA. ${ }^{15}$

\section{Systematic literature review}

The bibliographic databases Cochrane, Embase, PsycINFo, PubMed, Scopus and Web of Science were searched with the name of one or more of the diseases of interest in the title and the word 'pain' and a word referring to a mode of intervention or care in the title, abstract or keywords (search date: 19 October 2015). For efficiency in answering the broad question of the literature review and to benefit from the work that was done previously, the search was limited to systematic reviews, meta-analyses, (practice) guidelines and recommendations. If no systematic reviews were available, we searched for RCTs. No time or language restrictions were applied in the initial search. Thus, included in the literature search were systematic reviews in one of the selected diseases (RA, SpA, PsA, OA) with pain as an outcome measure. Excluded were studies involving general pharmacological and joint-specific medical and surgical treatment.
All abstracts were independently read and judged on their suitability for inclusion by two reviewers. Results were compared and, in case of discrepancy, discussed until consensus was reached. Excluded were duplicate articles, articles that were withdrawn, those not written in English, animal studies, conference abstracts, articles including (practice) guidelines or recommendations without a systematic review or meta-analysis included, previous versions of reviews and meta-analyses (eg, Cochrane reviews), articles that did not have pain as a reported outcome measure or did not report outcomes for OA, RA, SpA or PsA, articles not reviewing the effect of one or multiple modes of intervention or care, and articles that only reviewed the effects of pharmacological treatments, surgical treatments, complementary medicine, herbs or nutraceuticals. Reference lists of the selected articles were hand-searched for additional relevant systematic reviews and meta-analyses. The detailed search keys and exclusion criteria are shown in online supplementary file 1 .

\section{Evaluating the evidence}

The evidence for OA was divided into evidence for OA in general; OA of the knee, hip or knee and hip; OA of the hand/ wrist and OA of the foot/ankle. The systematic reviews and meta-analyses commonly included a mean effect size for pain. For every treatment option and per disease subgroup, the effect found by the included articles was recorded and effect categories were distinguished: 'positive effect' (ie, articles (unanimously) state positive effects of the treatment option on pain), 'no effect' (ie, articles state the treatment option has neither positive nor negative effects), 'unclear effect' (ie, articles state both no effects and positive effects) or a combination thereof meaning that articles were divided in their conclusions on the effect of the treatment option (eg, some state unclear effects and others only positive effects). 'Negative effect' could have been a category but none of the included studies stated harmful overall effects of the examined treatment options.

Our systematic review protocol was developed as a review of reviews including systematic reviews and meta-analyses of RCTs. Therefore, the Oxford Centre for evidence-based medicine 'level of evidence' for all recommendations was 1A (from meta-analysis or RCTs) or occasionally 1B (when only one RCT was available). ${ }^{22}$ The Grades of Recommendation, Assessment, Development and Evaluation system was used to rate the overall quality of evidence of the reviews and meta-analyses. ${ }^{32}$ Two assessors independently graded the quality of the available evidence as high, moderate, low or very low. In case of discrepancy, the quality was discussed until consensus was reached. ${ }^{33}$ 'Strength of recommendation' was determined for the recommendations. These scores vary from A ('category of evidence' 1A: meta-analysis of RCTs) to D ('category of evidence' 4 from expert committee reports or opinions and/or clinical experience of respected authorities or extrapolated recommendation from 'category of evidence' 2 or 3 from non-randomised experimental, correlation or descriptive studies). ${ }^{22}$

\section{Developing recommendations}

During the second and last task force meeting, the results of the systematic literature were presented and discussed, and the wording of recommendations was started. Treatment recommendations were supported by findings in the systematic literature review. Overarching principles and assessment recommendations were mostly based on expert opinion in the task force. After this meeting, the wording was finished through email, and each task force member indicated the 'level of agreement' on a numerical 


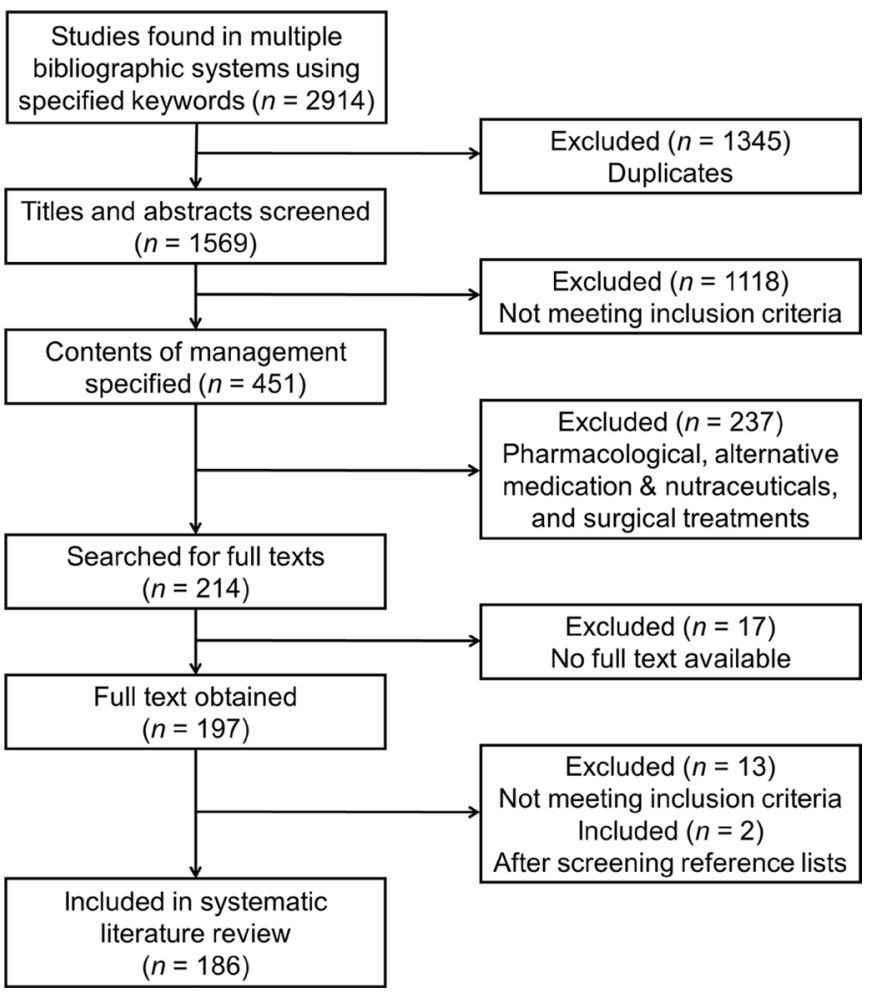

Figure 1 Flow chart of the systematic literature review of systematic reviews.

rating scale ranging from 0 (completely disagree) to 10 (completely agree).

\section{RESULTS}

Figure 1 shows a flow chart of the systematic literature review. The 2914 selected titles reduced to 214 after exclusion of duplicates and check of exclusion criteria. Full texts of 17 articles could not be obtained. Two assessors read 197 articles in full-text. Another 13 articles were excluded for being a narrative (non-systematic) review $(3 \mathrm{x})$, guideline without systematic review $(1 \mathrm{x})$ or duplicate $(2 x)$, not having pain as an outcome $(4 x)$ or treatment effects on pain as an aim $(2 \mathrm{x})$ or involving only results of pharmacological treatment $(1 \mathrm{x})$. Two additional meta-analyses were included after scanning the reference list of the selected articles. In total, 186 systematic reviews and meta-analyses were included, and their content was assessed for the following information: population, types of intervention, effects and level of quality. This information is provided in online supplementary files 2, 3 and 4 for all included studies.

\section{Evaluation of effects on pain (systematic literature review)}

No studies were found that systematically reviewed effects on pain for PsA. Moreover, it was found that of the included treatment options, sleep interventions and most assistive devices were not evaluated for their effect on pain in systematic reviews. Studies were heterogeneous with respect to intervention and comparator conditions. For example, comparisons of exercise included land-based versus water-based, strengthening versus aerobic, group versus individual, supervised versus home-based, multimodal versus unimodal, progressive versus non-progressive and one or various exercises versus standard care, sham or medication. The reviewed studies, direction of effects and level of quality are shown in tables 1 and 2 for the treatment modalities
Table 1 Overview of systematic reviews of randomised trials (evidence category $1 \mathrm{~A}$ ) regarding education, orthotics, psychological interventions, weight management and multimodal treatment: treatment modality and disease, direction of effect and the quality of the evidence according to GRADE in patients with RA, SpA and OA

\begin{tabular}{|c|c|c|c|}
\hline $\begin{array}{l}\text { Treatment modality } \\
\text { Disease } \\
\text { Specific treatment modality }\end{array}$ & $\begin{array}{l}\text { Reviews } \\
\text { (n) }\end{array}$ & $\begin{array}{l}\text { Direction } \\
\text { of effect }\end{array}$ & $\begin{array}{l}\text { GRADE } \\
\text { quality of } \\
\text { evidence }\end{array}$ \\
\hline \multicolumn{4}{|l|}{ Education and self-management } \\
\hline RA & 8 & $0 /+$ & $\oplus \oplus$ \\
\hline SpA & 1 & 0 & $\oplus \oplus$ \\
\hline OA-general & 6 & $0 /+$ & $\oplus \oplus \oplus$ \\
\hline OA-hand/wrist & 1 & 0 & $\oplus$ \\
\hline OA-hip/knee & 4 & + & $\oplus \oplus \oplus$ \\
\hline OA-knee & 4 & + & $\oplus \oplus \oplus$ \\
\hline \multicolumn{4}{|l|}{ Orthotics } \\
\hline \multicolumn{4}{|l|}{ RA } \\
\hline Orthotic gloves & 2 & $0 /+$ & $\oplus \oplus$ \\
\hline Splints & 5 & $0 /+$ & $\oplus \oplus$ \\
\hline Insoles & 8 & $0 /+$ & $\oplus \oplus$ \\
\hline Orthopaedic shoes & 3 & + & $\oplus \oplus$ \\
\hline Padded hosiery & 1 & + & $\oplus$ \\
\hline \multicolumn{4}{|l|}{ OA-hand/wrist } \\
\hline Orthotic gloves & 1 & 0 & $\oplus$ \\
\hline Splints & 8 & + & $\oplus \oplus$ \\
\hline \multicolumn{4}{|l|}{ OA hip } \\
\hline Insoles & 1 & + & $\oplus$ \\
\hline \multicolumn{4}{|l|}{ OA-knee } \\
\hline Braces & 10 & $? /+$ & $\oplus \oplus$ \\
\hline Sleeves & 1 & + & $\oplus \oplus$ \\
\hline Elastic bandages & 2 & + & $\oplus \oplus$ \\
\hline Taping & 3 & $? /+$ & $\oplus \oplus$ \\
\hline Orthoses in general & 1 & + & $\oplus \oplus$ \\
\hline Insoles & 15 & $? /+$ & $\oplus \oplus$ \\
\hline Orthopaedic shoes & 1 & + & $\oplus \oplus$ \\
\hline Cane & 1 & + & $\oplus \oplus$ \\
\hline \multicolumn{4}{|l|}{ Psychological interventions } \\
\hline \multicolumn{4}{|l|}{ RA } \\
\hline Cognitive-behavioural therapy & 7 & + & $\oplus \oplus \oplus$ \\
\hline Biofeedback & 1 & + & $\oplus \oplus$ \\
\hline \multicolumn{4}{|l|}{ SpA } \\
\hline Cognitive-behavioural therapy & 1 & 0 & $\oplus$ \\
\hline \multicolumn{4}{|l|}{ OA-general } \\
\hline Cognitive-behavioural therapy & 1 & + & $\oplus \oplus \oplus$ \\
\hline Psychosocial and coping interventions & 1 & + & $\oplus \oplus \oplus$ \\
\hline Relaxation techniques & 1 & + & $\oplus$ \\
\hline \multicolumn{4}{|l|}{ OA-hip/knee } \\
\hline Relaxation techniques & 1 & + & $\oplus$ \\
\hline \multicolumn{4}{|l|}{ OA-knee } \\
\hline Biofeedback & 1 & 0 & $\oplus$ \\
\hline \multicolumn{4}{|l|}{ Weight management } \\
\hline RA & 2 & + & $\oplus \oplus$ \\
\hline SpA & 1 & + & $\oplus$ \\
\hline OA-hip/knee & 2 & + & $\oplus \oplus \oplus$ \\
\hline OA-knee & 10 & $\mathrm{O} /+$ & $\oplus \oplus \oplus$ \\
\hline
\end{tabular}

Multimodal treatment

RA
$\begin{aligned} & \text { Comprehensive occupational therapy } \\ & \text { OA-hand/wrist }\end{aligned}$
$\begin{array}{llll}\text { Multidisciplinary therapy } & 1 & 0 & \oplus \oplus \\ \end{array}$




\begin{tabular}{llll}
\hline Table 1 Continued & & & \\
\hline $\begin{array}{l}\text { Treatment modality } \\
\text { Disease }\end{array}$ & $\begin{array}{l}\text { Reviews } \\
(\mathbf{n})\end{array}$ & $\begin{array}{l}\text { Direction } \\
\text { of effect }\end{array}$ & $\begin{array}{l}\text { GRADE } \\
\text { quality of } \\
\text { evidence }\end{array}$ \\
\hline $\begin{array}{c}\text { Specific treatment modality } \\
\text { OA knee }\end{array}$ & 1 & 0 & $\oplus \oplus \oplus$ \\
\hline Comprehensive physical therapy & 1 & & \\
\hline
\end{tabular}

Diseases without a review were excluded from the table.

Direction of effect:+, positive; 0 , no; -, negative; ?, unclear (effect equivocal), or a

combination thereof meaning that different reviews reached different conclusions about the effect of the treatment.

References are shown in online supplementary file 2.

GRADE, $\oplus \oplus \oplus \oplus$, high; $\oplus \oplus \oplus$, moderate; $\oplus \oplus$, low; $\oplus$, very low.

GRADE, Grades of Recommendation, Assessment, Development and Evaluation; OA

osteoarthritis; RA, rheumatoid arthritis; SpA, spondyloarthritis.

Table 2 Overview of systematic reviews of randomised trials (evidence category $1 \mathrm{~A}$ ) regarding 'physical activity and exercise': treatment modality and disease, direction of effect and the quality of the evidence according to GRADE in patients with RA, SpA and OA

\begin{tabular}{|c|c|c|c|}
\hline $\begin{array}{l}\text { Treatment modality } \\
\text { Disease }\end{array}$ & Reviews (n) & Direction of effect & $\begin{array}{l}\text { GRADE quality } \\
\text { of evidence }\end{array}$ \\
\hline \multicolumn{4}{|l|}{ General exercise } \\
\hline RA & 5 & $0 /+$ & $\oplus \oplus$ \\
\hline SpA & 6 & + & $\oplus \oplus$ \\
\hline OA-general & 6 & + & $\oplus \oplus \oplus$ \\
\hline OA-hand/wrist & 4 & $0 /+$ & $\oplus \oplus$ \\
\hline OA-hip/knee & 11 & + & $\oplus \oplus \oplus$ \\
\hline OA-hip & 11 & o/+ & $\oplus \oplus$ \\
\hline OA-knee & 18 & + & $\oplus \oplus \oplus$ \\
\hline OA-foot/ankle & 2 & + & $\oplus \oplus$ \\
\hline \multicolumn{4}{|l|}{ Aerobic exercise } \\
\hline RA & 3 & o/+ & $\oplus \oplus$ \\
\hline OA-general & 3 & + & $\oplus \oplus \oplus$ \\
\hline OA-hip/knee & 2 & o/t+ & $\oplus \oplus$ \\
\hline OA-hip & 1 & 0 & $\oplus$ \\
\hline OA-knee & 9 & + & $\oplus \oplus \oplus$ \\
\hline \multicolumn{4}{|c|}{ Strength and resistance } \\
\hline RA & 2 & o/+ & $\oplus \oplus$ \\
\hline OA-general & 3 & + & $\oplus \oplus \oplus$ \\
\hline OA-hand/wrist & 2 & $0 /+$ & $\oplus \oplus$ \\
\hline OA-hip/knee & 4 & + & $\oplus \oplus \oplus$ \\
\hline OA-hip & 3 & + & $\oplus \oplus$ \\
\hline OA-knee & 14 & + & $\oplus \oplus \oplus$ \\
\hline \multicolumn{4}{|c|}{ Tai chi, yoga, qigong, whole body vibration } \\
\hline RA & 3 & $? /+$ & $\oplus$ \\
\hline OA-general & 6 & o/+ & $\oplus$ to $\oplus \oplus$ \\
\hline OA-hand/wrist & 3 & + & $\oplus$ \\
\hline OA-hip/knee & 1 & o/+ & $\oplus \oplus$ \\
\hline OA-knee & 12 & $0 /+$ & $\oplus$ to $\oplus \oplus$ \\
\hline
\end{tabular}

Diseases without a review were excluded from the table.

Direction of effect: + , positive; 0 , no; - , negative; ? unclear (effect equivocal) or a

combination thereof meaning that different reviews reached different conclusions about the effect of the treatment.

References are shown in online supplementary file 3 .

GRADE: $\oplus \oplus \oplus \oplus$, high; $\oplus \oplus \oplus$, moderate; $\oplus \oplus$, low; $\oplus$ to $\oplus \oplus$, very low to low; $\oplus$, very low. (The combined $\oplus$ to $\oplus \oplus$ grade is due to difference in quality between studies of different modalities.

GRADE, Grades of Recommendation, Assessment, Development and Evaluation; OA osteoarthritis; RA, rheumatoid arthritis; SpA, spondyloarthritis.
Table 3 Review of reviews (evidence category $1 \mathrm{~A}$ ) regarding miscellaneous therapies: treatment modality and disease, direction of effect and the quality of the evidence according to GRADE in patients with RA, SpA and OA

\begin{tabular}{|c|c|c|c|}
\hline $\begin{array}{l}\text { Treatment modality } \\
\text { Disease }\end{array}$ & Reviews ( $n$ ) & $\begin{array}{l}\text { Direction of } \\
\text { effect }\end{array}$ & $\begin{array}{l}\text { GRADE quality } \\
\text { of evidence }\end{array}$ \\
\hline \multicolumn{4}{|l|}{ Acupuncture } \\
\hline RA & 5 & $0 /+$ & $\oplus \oplus$ \\
\hline OA-general & 4 & $\mathrm{ol}+$ & $\oplus \oplus$ \\
\hline OA-hand/wrist & 3 & o/+ & $\oplus$ \\
\hline OA-hip/knee & 2 & + & $\oplus \oplus$ \\
\hline OA-hip & 1 & 0 & $\oplus \oplus$ \\
\hline OA-knee & 16 & + & $\oplus \oplus \oplus$ \\
\hline \multicolumn{4}{|l|}{ Balneotherapy and massage } \\
\hline RA & 3 & o/t+ & $\oplus \oplus$ \\
\hline SpA & 2 & $\mathrm{o} /+$ & $\oplus$ \\
\hline OA-general & 5 & $\mathrm{o} /+$ & $\oplus$ to $\oplus \oplus$ \\
\hline OA-hand/wrist & 3 & + & $\oplus$ \\
\hline OA-hip/knee & 2 & o/t+ & $\oplus$ \\
\hline OA-knee & 8 & + & $\oplus \oplus$ \\
\hline \multicolumn{4}{|l|}{ Thermotherapy } \\
\hline RA & 4 & $0 /+$ & $\oplus \oplus$ \\
\hline OA-general & 1 & 0 & $\oplus$ \\
\hline OA-hand/wrist & 3 & $0 /+$ & $\oplus \oplus$ \\
\hline OA-knee & 4 & $0 /+$ & $\oplus \oplus$ \\
\hline \multicolumn{4}{|l|}{$\begin{array}{l}\text { Ultrasound, radiotherapy and } \\
\text { diathermy }\end{array}$} \\
\hline RA & 3 & + & $\oplus \oplus$ \\
\hline OA-general & 2 & $0 /+$ & $\oplus \oplus$ \\
\hline OA-hip/knee & 2 & $? / 0$ & $\oplus \oplus$ \\
\hline OA-knee & 12 & $0 /+$ & $\oplus \oplus$ to $\oplus \oplus \oplus$ \\
\hline \multicolumn{4}{|l|}{ Electromagnetic therapy } \\
\hline RA & 2 & $\mathrm{o} /+$ & $\oplus \oplus$ \\
\hline OA-general & 1 & + & $\oplus \oplus \oplus$ \\
\hline OA-hip/knee & 3 & + & $\oplus \oplus$ \\
\hline OA-knee & 18 & $? /+$ & $\oplus \oplus$ to $\oplus \oplus \oplus$ \\
\hline \multicolumn{4}{|l|}{ Laser therapy } \\
\hline RA & 3 & + & $\oplus \oplus$ \\
\hline OA-general & 2 & o/+ & $\oplus \oplus$ \\
\hline OA-hand/wrist & 4 & 0 & $\oplus \oplus$ \\
\hline OA-knee & 7 & $0 /+$ & $\oplus \oplus \oplus$ \\
\hline \multicolumn{4}{|l|}{ Magnet therapy } \\
\hline OA-general & 1 & o/t+ & $\oplus \oplus$ \\
\hline OA-hand/wrist & 2 & $0 /+$ & $\oplus \oplus$ \\
\hline OA-knee & 2 & 0 & $\oplus \oplus$ \\
\hline \multicolumn{4}{|c|}{ Manual therapy/joint mobilisation } \\
\hline OA-hand/wrist & 4 & + & $\oplus \oplus \oplus$ \\
\hline OA-hip/knee & 1 & $0 /+$ & $\oplus \oplus$ \\
\hline OA-hip & 1 & + & $\oplus \oplus$ \\
\hline OA-knee & 1 & + & $\oplus \oplus$ \\
\hline \multicolumn{4}{|l|}{ Diverse } \\
\hline $\begin{array}{l}\text { OA-general (healing, qigong, } \\
\text { chiropractic) }\end{array}$ & 1 & $\mathrm{O} /+$ & $\oplus$ \\
\hline $\begin{array}{l}\text { OA-hand/wrist (leeches, copper } \\
\text { bracelets) }\end{array}$ & 2 & $\mathrm{o} / ?$ & $\oplus$ \\
\hline
\end{tabular}

Diseases without a review were excluded for the table.

Direction of effect: +, positive; 0, no; - , negative; ?, unclear (effect equivocal) or a combination thereof meaning that different reviews reached different conclusions about the effect of the treatment or that the direction of effects differed between treatment modalities such as between transcutaneous electrical nerve stimulation and pulsed electromagnetic field therapy which are both included in 'electromagnetic therapy'.

References are shown in online supplementary file 4.

GRADE: $\oplus \oplus \oplus \oplus$, high; $\oplus \oplus \oplus$, moderate; $\oplus \oplus$ to $\oplus \oplus \oplus$, low to moderate; $\oplus \oplus$, low; $\oplus$ to $\oplus \oplus$, very low to low; $\oplus$ very low. (The combined grades are due to difference in quality between studies of different modalities such as balneotherapy vs massage studies.) GRADE, Grades of Recommendation, Assessment, Development and Evaluation; OA osteoarthritis; RA, rheumatoid arthritis; SpA, spondyloarthritis. 
that were included in the recommendations and in table 3 for miscellaneous treatment modalities.

\section{Education and self-management}

The reviewed review studies on education and self-management programmes generally concluded that available studies showed 'positive effects' $(n=8)$ or showed both 'no effects' and 'positive effects' $(n=14)$, but for SpA and OA of the hand/wrist, single meta-analyses observed 'no effect' on pain (table 1).

\section{Physical activity and exercise}

Of all treatment options, the effects of physical exercise have been studied most extensively (table 2). For general exercise, aerobic exercise, and strength and resistance training, the quality of studies was low to moderate and effects on pain were mostly 'positive' in IA and OA, with some reviews observing 'no effects'. For tai chi, yoga, qigong and whole body vibration, the quality of studies was low to very low, and it was unclear whether there were positive effects on pain. Table 2 can be used as a guide for choosing an appropriate intervention; for instance, strength and resistance training is more relevant and more extensively studied for OA of the knee than for other conditions. Reviews do not answer the question whether high-intensity exercise is as safe as low-intensity exercise which is an ongoing issue of debate. ${ }^{34} 35$

\section{Orthotics}

In mostly low quality studies, 'positive effects' of orthotics on pain have been consistently observed for orthopaedic shoes in RA and OA of the knee, splints in OA of the hand, and sleeves and elastic bandages in OA of the knee and less consistently for other orthotics (table 1). Except for use of a cane, ${ }^{16}$ no systematic reviews evaluated daily living aids such as a tin-opener or assistive devices using pain as an outcome. Although several orthotics can be recommended based on positive effects on pain, there is not enough evidence to give recommendations regarding design or materials.

\section{Psychological and social interventions}

In very low to moderate quality studies, effects of psychological interventions (eg, cognitive-behavioural therapy (CBT), mindfulness-based interventions, stress management training) on pain as summarised in reviews were 'positive' with the exception of reviews showing 'no effect' on pain of CBT in SpA and biofeedback in OA (table 1).

\section{Weight management}

In very low to moderate quality studies, effects of weight management have been frequently reviewed for OA of the knee; 'no effects' and 'positive effects' were observed. For RA and SpA, 'positive effects' were observed in three reviews (table 1).

\section{Sleep interventions}

It has been proposed that sleep disturbance should be systematically assessed and managed in patients with IA and OA. ${ }^{13}$ 36-38 Our systematic review did not extract systematic reviews that evaluated effects of sleep interventions on pain in OA or IA, but randomised trials examined the effects of CBT for insomnia in OA. CBT was observed to improve sleep and pain in one study. ${ }^{39}$ In another study, both CBT and a placebo condition resulted in improved sleep and comparable reductions of pain over 6 months, but the CBT group had significantly greater reductions in wake after sleep onset which predicted subsequent decreases in clinical pain. ${ }^{40}$ Outside the field of rheumatic diseases, meta-analyses support the effectiveness of behavioural, including self-help, interventions on sleep outcomes. ${ }^{41} 42$ Face-to-face treatments of at least four sessions seem to be more effective than self-help interventions. ${ }^{43}$ In meta-analyses, small but significant effects of sleep interventions on pain have been observed in people with varied chronic medical conditions. ${ }^{44} 45$

\section{Pharmacological treatment}

Pharmacological treatment is a core ingredient of pain management in IA and OA. It includes analgesics (eg, paracetamol, codeine and other opiate-like drugs); oral or topical non-steroidal anti-inflammatory drugs (NSAIDs); intra-articular injections, for example, with glucocorticoids; and occasionally also agents for neuropathic pain. The evidence for pharmacological pain treatment was not part of the current review but has been evaluated by other task forces. ${ }^{16}{ }^{17} 24-294^{46-50}$ In brief, previous task forces recommended paracetamol as first-line treatment, with topical agents such as topical NSAIDs and capsaicin also recommended for specific joints, for patients with OA, ${ }^{172629}$ and consideration of intra-articular injections for specific joints in OA and IA. ${ }^{17} 24-262829$ Existing EULAR recommendations should be consulted regarding the safe use of NSAIDs. ${ }^{17262946}$

\section{Miscellaneous therapies}

Table 3 shows an overview of less commonly available therapies in rheumatological clinical practice that were therefore not included in the recommendations. If a patient shows interest in one of these therapies, and skilled professionals are available to administer them, then this overview can be used as a quick guide to the appropriate meta-analyses. These therapies have especially been studied in OA of the knee with positive effects on pain of acupuncture and 'balneotherapy and massage' and less clear effects of 'electrical' therapies.

\section{Psoriatic arthritis}

Pain is as high or higher in PsA than in RA, ${ }^{51}$ and patients have an educational need to manage their pain. ${ }^{52}$ Nevertheless, none of the extracted studies reviewed the health professional's approach in PsA for effects on pain. Given the lack of specific knowledge, the health professional may use pain treatment options in RA to guide pain treatment in PsA.

\section{Overarching principles (expert opinion)}

The task force defined overarching principles based on expert opinion (box 1).

First, patient-centred care was considered important. Care that is respectful of and responsive to individual patient preferences, needs and values, and ensures that patient values guide clinical decisions, ${ }^{53}$ may improve adherence and persistence with treatment. $^{21}{ }^{54-57}$ Validation of the patient's pain experience is considered a prerequisite for trust, communication and engagement in treatment.

Second, a biopsychosocial model of pain was recommended. Relationships between all factors of this model are recognised to be interactive and reciprocal with mutually influencing pathways similar to a hanging mobile toy in which movement of one part causes movement of other parts. The importance of the distinct factors differs between individuals.

Third, in order to achieve pain control, it is crucial to treat disease activity and to prevent tissue damage. To meet that aim, the health professional should have basic knowledge of IA and OA. Common treatment goals in rheumatic diseases are to optimise control of inflammation, decrease disease activity, improve 


\section{Box 1 Overarching principles}

- The assessment and treatment process should be guided by a patient-centred framework.

- The health professional should understand that (any type of) pain encompasses multiple and mutually interacting biological, psychological and social factors that include but are not limited to pain severity, peripheral (inflammation and joint damage) and central neurophysiological processes, physical (dis)ability, resilience and vulnerabilities (emotions, cognitions, behaviour, lifestyle), social factors (work, support, facilities, economic), sleep quality, obesity and other health risks (eg, smoking, alcoholism).

- The health professional should have basic knowledge of the pathology, treatment and sequelae of inflammatory arthritis and osteoarthritis.

- The health professional should be able to differentiate between localised and generalised pain and should know that these types of pain may coexist.

function and well-being, and to reduce pain and other disease-related symptoms. ${ }^{15-17} 2124-29$

Fourth, the ability to differentiate between types of pain helps the health professional to direct the optimal pain-management strategy. Pain localised in a specific region of the body might be due to peripheral nociceptive input such as inflammation or damage. Generalised pain is more often non-specific with regard to pathological findings and can be due to a dysfunction in the regulation of pain pathways. Pain is commonly regarded as generalised or widespread when pain is present in both sides of the body, above and below the waist and in axial body regions. In IA, generalised pain may remain despite good inflammatory control. Such pain requires comprehensive pain-management strategies. $^{5859}$

Not all health professionals may currently have the required knowledge and skills to apply these principles. For those health professionals who have identified that they require further education in this area, the overarching principles can be used to direct their learning. This may involve work-based supervision with another health professional or undertaking an educational course that addresses some or all of these principles.

\section{Recommendations (systematic literature review and expert opinion)}

Table 4 shows the recommendations. Proper assessment is a prerequisite for proper pain treatment. Recommendations 1 (assessment) and 2 (personalised treatment plan) were based on expert opinion in the task force. Treatment recommendations 3-10 were based on the systematic literature review summarising effects on pain. Specific considerations regarding application of these treatment options (indicated with an asterisk in table 4) were based on expert opinion of the task force.

\section{Assessment}

The extent of assessment depends on many factors such as available time. A first step in ensuring that pain management is patient-centred is to invite patients disclosing the impact of pain on their daily functioning, to assess their ideas and concerns regarding the cause of their pain and the perceived control over pain episodes, and to take account of their expectations and preferences for treatment. It is deemed important to establish the patient's functional and valued life goals, that is, what it is that they cannot currently do as well as they would wish to. Research shows that individuals differ widely in terms of management needs. ${ }^{21546061}$

Second, assess pain severity using a numerical or visual analogue pain rating scale, ${ }^{62}$ and the onset, duration, location and spread (pain manikin), quality, interference, triggers and progression of pain. Furthermore, appraise the type of pain (localised or generalised) and whether referral is needed to a pain specialist to evaluate the type of pain, current treatment or current medication (safe use, interactions with other medication, side effects). Generalised pain can be recognised in a clinical interview and by the use of a pain manikin such as the Michigan body map. ${ }^{63}$ Use validated questionnaires to assess the potential presence of neuropathic pain. ${ }^{6264-66}$

Third, assess ongoing pharmacological and non-pharmacological treatments, previous treatments tried and the effects and side effects of these treatments, patient beliefs about the ability to control and overcome pain and its consequences, and willingness of the patient to engage in additional treatment if deemed necessary.

Fourth, assess current inflammation and joint damage as sources of pain following the most recent recommendations. ${ }^{6768}$ In case of poorly controlled inflammation, optimise disease control or refer to a rheumatologist for treatment according to recommendations. ${ }^{252748}$ In case of localised (nociceptive) pain relating to OA, consider (to refer to) joint-specific treatments in line with recommendations. ${ }^{16} 69$

Fifth, assess pain-related biological, psychological and social factors that might need attention, specifically:

- The nature and extent of disability: physical activity, mobility, activities of daily living, social participation, general physical fitness (aerobic capacity, muscle strength, endurance), pain-related fear and avoidance of activities, balance of activities and rest (pacing).

- Beliefs and emotions about pain and pain-related disability: the psychological response to pain and psychological vulnerability factors, psychological distress, psychiatric comorbidity and cognitions such as catastrophising (rumination, magnification and helplessness), ${ }^{70}$ fear of movement-related pain, ${ }^{71}$ catastrophising and pain self-efficacy. ${ }^{72}$

- Social factors related to pain and its consequences: the way family members and other significant others react to patient's pain or pain-related disability; work; family and friends; economic problems; housing.

- Sleep problems: the quantity and quality of sleep, including whether the patient feels refreshed on waking and sleep hygiene habits such as regular exercise during the day, stress management, noise, sleep timing and avoidance of caffeine, nicotine, alcohol and daytime napping. ${ }^{73}$

- Presence of obesity. ${ }^{74}$

- Other factors that might influence pain or pain management, such as dependence on tobacco, alcohol or drugs. ${ }^{75}$

\section{Treatment}

Tables 1-3 offer an overview of the number of the reviewed reviews and meta-analyses, the observed effects of specific treatments on pain and the quality of evidence of the studies.

Through shared decision-making, treatment is guided by the expressed needs of the patient, the health professional's assessment and evidence-based treatment options. A stepped-care approach is recommended including education and self-management support in step 1 (recommendation 3), one or more treatment options by a specialist if indicated in step 2 (recommendations 4-9) or multidisciplinary treatment in step 3 (recommendation 10). 
Table 4 EULAR recommendations for the health professionals' approach to pain management in inflammatory arthritis and osteoarthritis

\begin{tabular}{|c|c|c|c|}
\hline & $\begin{array}{l}\text { Level of } \\
\text { evidence }\end{array}$ & $\begin{array}{l}\text { Strength of } \\
\text { recommendation }\end{array}$ & $\begin{array}{l}\text { Level of agreement } \\
\text { task force: mean (SD) }\end{array}$ \\
\hline $\begin{array}{l}\text { 1. Assessment by the health professional should include the following aspects (the assessment is brief or extensive depending } \\
\text { on factors such as available time, whether it is a first or regular consultation, and the needs of the patient): }\end{array}$ & 4 & D & $9.3(0.8)$ \\
\hline \multicolumn{4}{|l|}{ Patient's needs, preferences and priorities regarding pain management and important activities, values and goals in daily life. } \\
\hline \multicolumn{4}{|l|}{ Patient's pain characteristics including severity, type, spread and quality. } \\
\hline \multicolumn{4}{|l|}{ Previous and ongoing pain treatments and the perceived efficacy. } \\
\hline Current inflammation and joint damage as sources of pain, and whether these are adequately treated. & & & \\
\hline
\end{tabular}

2. The patient should receive a personalised management plan with the aim of reducing pain and pain-related distress and improving pain-related function and participation in daily life. This plan is guided by shared decision-making, the expressed

4

D

$9.0(0.8)$

needs of the patient, the health professional's assessment and evidence-based treatment options. A stepped-care approach may include, in step 1, education and self-management support (recommendation 3); in step 2, one or more treatment options by a specialist if indicated (recommendations 4 to 9); or, in step 3, multidisciplinary treatment (recommendation 10).

3. The patient should receive education.

${ }^{*}$ All patients have easy access to (1) educational materials (such as brochures or links to online resources with

encouragement to stay active, sleep hygiene guidelines and so on), (2) psychoeducation by the health professional and (3) online or face-to-face self-management interventions.

4. If indicated, the patient should receive physical activity and exercise.

* The health professional and patient appraise whether advice to stay active, supervised physical exercise or multidisciplinary treatment is needed.

* If the patient is not able to initiate physical activity and exercises without help, then consider the possibility for referral to a physiotherapist for individually tailored graded physical exercise or strength training.

* If psychosocial factors such as fear of movement ${ }^{7180}$ or catastrophising cognitions ${ }^{70}$ underlie a disabled, sedentary lifestyle, then consider a multidisciplinary intervention including cognitive - behavioural therapy.

5. If indicated, the patient should receive orthotics.

* If a patient has pain during activities of daily living which impedes functioning, orthotics (such as splints, braces, gloves, sleeves, insoles and shoes), daily living aids (such as a tin opener), an assistive device (such as a cane or rollator) or ergonomic adaptation (at home, workplace) can be offered. If the patients wants to use this assistive support, then consider referral to the occupational therapist, who can proceed with several actions: offer education about appropriate ways to use joints and ergonomic principles, appraise the need for the use of an orthotic or assistive device, give advice about how to acquire it, fit the customised aid to the patient, offer training in the use of it, refer to the appropriate specialist who will do this, eg, orthopaedic shoemaker.

6. If indicated, the patient should receive psychological or social interventions.

* If there are indications that social variables or psychological factors interfere with effective pain management and

functional status, then consider (depending on the severity) providing basic social and psychological management support or referral to a psychologist, social worker, self-management support programme, CBT or multidisciplinary treatment.

* If psychopathology (eg, depression and anxiety) is present, discuss treatment options with the patient and the patient 's primary care physician.

7. If indicated, the patient should receive sleep interventions.

* If sleep disturbance is reported, inquire about causes (eg, pain, persistent worrying, poor sleep habits) and offer basic education about good sleep hygiene practices.

* If sleep remains (severely) disturbed, refer to a therapist or programme aimed at restoring sleep, or to a specialised sleep clinic.

8. If indicated, the patient should receive weight management.

* If the patient is obese, explain to the patient that obesity can contribute to pain and disability. Discuss accessible weight management options with the patient or signpost appropriate specialised weight management support; for example, dietitian , psychologist, community lifestyle services or bariatric clinic/surgery.

9. If indicated, the patient should receive pharmacological and joint-specific pain treatment according to recent recommendations.

${ }^{*}$ Ask about the patient 's existing use of prescribed and over - the - counter pain relief including homeopathic remedies and consider if the frequency of use is safe (not over dosing) and appropriately regular. Ask or refer for further specialist or medical advice if there are concerns or if additional pharmacological treatment may be indicated.

10. If indicated, the patient should receive multidisciplinary treatment.

* If more than one treatment options are indicated, for example, to treat psychological distress in combination with a sedentary lifestyle, and if monotherapy failed, consider a multidisciplinary intervention.

'Level of evidence' and 'Strength of recommendation' for treatment modalities refer to specific diseases in which uniform positive effects on pain (excluding studies with 'very low' quality of evidence) were observed (tables 1 and 2). Overarching principles and recommendations regarding assessment are based on expert opinion.

Level of evidence: $1 \mathrm{~A}$, from meta-analysis of randomised controlled trials; $1 \mathrm{~B}$, from at least one randomised controlled trial; $2 \mathrm{~A}$, from at least one controlled study without randomisation;

$2 \mathrm{~B}$, from at least one other type of quasi-experimental study; 3 , from descriptive studies, such as comparative studies, correlation studies or case-control studies; 4 , from expert committee reports or opinions and/or clinical experience of respected authorities.

Strength of recommendations is a combination of the information from the systematic literature review and expert opinion: $A$, category I evidence; $B$, category II evidence or extrapolated recommendations from category I evidence; C, category III evidence or extrapolated recommendation from category I or II evidence; D, category IV evidence or extrapolated recommendation from category II or III evidence. 22

Level of agreement by the task force on a scale from 0 to 10.

*Specific considerations regarding application of recommendations that are indicated with an asterisk are based on expert opinion of the task force.

CBT, cognitive-behavioural therapy; EULAR, European League Against Rheumatism.

The choice for a specific intervention is not only determined by effects on pain but also by effects on functioning, social participation and well-being. Moreover, evidence for effects of specific pain treatments differ for specific diseases. The 'strength of recommendation' for recommendations 3-10 (table 4) holds for specific diseases in which uniform positive effects on pain (excluding studies with 'very low' quality of evidence) were observed. 


\section{Box 2 Research agenda}

- To examine omissions in knowledge such as effects of treatment options on pain in psoriatic arthritis, the effects of sleep interventions on pain in inflammatory arthritis (IA) and osteoarthritis (OA), and pain as an outcome measure in studies of assistive devices such as a cane or rollator in more diseases than $\mathrm{OA}$ of the knee alone.

- To examine in meta-analyses the effects of multidisciplinary treatment on pain.

- To improve the methodological quality of treatment outcome studies.

- To conduct an analysis examining effect sizes for more specific treatment modalities that are now merged into comprehensive treatment packages.

- To examine moderators of treatment effects (eg, in which patient subgroups each specific treatment option causes a reduction of pain).

- To examine mediators of outcome, that is, how pain treatments work in IA and OA.

- To examine the effects on pain of minimal interventions such as advice during a consultation, use of brochures and e-health psychoeducation.

- To examine whether combined pharmacological and non-pharmacological pain management is more effective than monotherapy.

- To contribute to personalised medicine by analysing customised pain treatments; for example, using replicated single-case experimental designs with idiosyncratic outcome measures.

- To examine in which way healthcare could best be organised to be able to provide the best possible and knowledgeable pain-management support for people with IA and OA.

- Education had a uniform positive effect on pain in OA (hip/ knee, knee).

- Physical activity and exercise showed uniform positive effects on pain for general exercise in SpA and OA (general, hip/knee, knee, foot/ankle), aerobic exercise in OA (general, knee), and strength and resistance training in OA (general, hip/knee, hip, knee).

- Orthotics showed small but consistent positive effects on pain for orthopaedic shoes in RA and OA of the knee, splints in $\mathrm{OA}$ of the hand and knee orthoses (especially sleeves, elastic bandages) in OA of the knee.

- Psychological and social interventions showed a uniform positive effect on pain for CBT in RA and OA (general), psychosocial and coping interventions in OA (general), biofeedback in RA and relaxation interventions in OA (general, hip/knee).

- There was no meta-analysis that evaluated effects of sleep interventions on pain in IA or OA but small effects of sleep interventions on pain were observed in meta-analyses in people with varied chronic medical conditions.

- Weight management showed a uniform positive effect on pain in RA, SpA and OA of the hip/knee.

- Multidisciplinary treatment is cautiously recommended considering the absence of studies examining the added effect on pain of multidisciplinary treatment to monodisciplinary therapies and considering that meta-analyses on multimodal treatment did not observe effects on pain.

\section{DISCUSSION}

Results and conclusions derived from 186 systematic reviews and meta-analyses were reviewed to identify the evidence associated with the benefits of the health professional's approach to pain management for people with IA and OA. Effects on pain were most uniformly positive for physical activity and exercise interventions, and for psychological interventions. Effects on pain for educational interventions, orthotics, weight management and multidisciplinary treatment were shown for particular disease groups. Recommendations for patient-centred pain management were guided by this scientific evidence and by clinical expert opinion.

The task force unanimously endorsed that in rheumatic care, besides pain severity, physical functioning and psychological functioning are major outcomes of any management intervention by health professionals, in agreement with other expert groups. ${ }^{3031}$ Although pain was selected as the target outcome, our systematic literature review also included interventions that were not aimed at alleviating pain but, for instance, at increasing muscle strength, physical activity or emotional functioning. Nevertheless, our study showed that many evaluations of treatment options-especially physical activity and psychological interventions-showed a reduction of pain in most patient groups. This included treatment options in which pain reduction was not the primary goal.

Box 2 presents questions for the future research agenda. Our systematic review showed that there is ample evidence for specific pain treatment options in specific groups. Nevertheless, there are several omissions in our knowledge with respect to effects of pain management on pain, especially in PsA, sleep interventions, assistive devices and multidisciplinary treatment. Moreover, it is inherent to the multifaceted nature of pain, the heterogeneity of the group of patients with IA and OA, and specific needs of individual patients that pain-management options of choice will differ among patients. From a clinical point of view, a multimodal approach will likely result in the best outcome, but from a scientific point of view, it would be more fruitful to learn whether a single treatment option is able to bring about change in pain and other outcomes. Thus, a main challenge in future research is to examine in which patient subgroups each specific treatment option causes a reduction of pain. Moreover, most studies pertain to systematic interventions in groups, but the most frequent clinical intervention is patient-customised education and advice given during a consultation, handing a brochure or offering information through the internet. The effects of these minimal interventions should be investigated as well.

As pain is the predominant symptom and burden in IA and $\mathrm{OA},{ }^{1-5}$ clinical training of rheumatology health professionals in pain management is essential. To ensure patient-centred pain management, health professionals need knowledge, confidence, communication skills and skills to support patients to translate intentions into action plans, which should be part of educational programmes. ${ }^{76-78}$ It has been demonstrated that training of professionals helps to improve pain management of OA. ${ }^{79}$ Health professionals can use the handout shown in figure 2 as a guide in their work, while the more detailed findings and recommendations can be used to fine-tune treatment. Further, knowledge and skills indicated in the overarching principles and recommendations should be used when reviewing pain curricula in higher education and in postgraduate clinical education. ${ }^{76}$ Within EULAR, the current recommendations can be included in the 'EULAR online course for health professionals' which comprises specific diseases such as RA and OA as well as broader modules: 


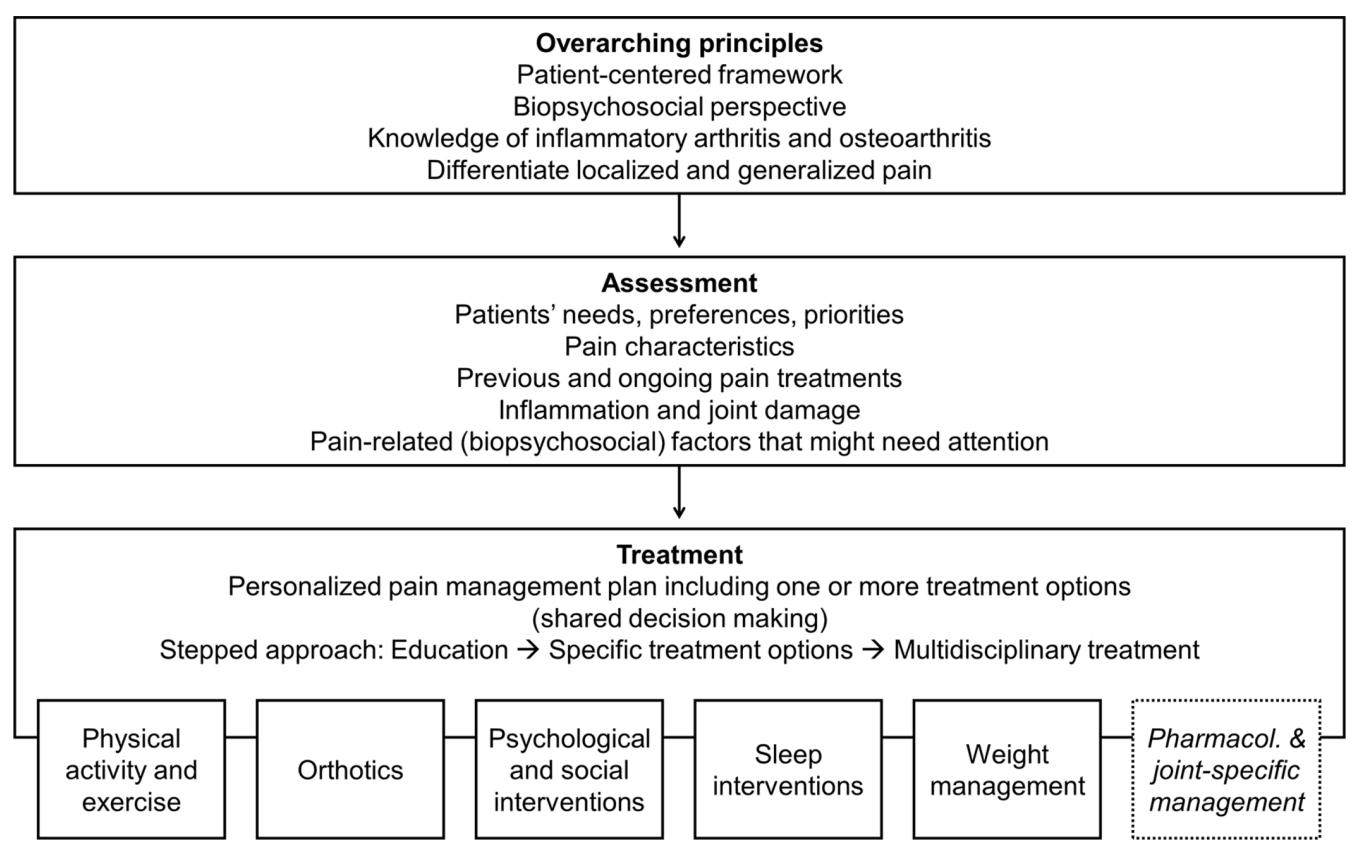

Figure 2 Handout with guide to pain management in inflammatory arthritis and osteoarthritis.

https://www.eular.org/edu online course hpr.cfm. Finally, these recommendations will be disseminated through this publication and through a lay summary of the recommendations that will be disseminated among national patient associations.

In conclusion, guided by expert opinion and partly underpinned by a considerable number of systematic reviews and meta-analyses, an expert group developed and launched the first set of recommendations that enable health professionals to provide knowledgeable and evidence-based pain-management support for people with IA and OA.

\section{Author affiliations}

${ }^{1}$ Department of Psychology, Utrecht University, Utrecht, The Netherlands ${ }^{2}$ Musculoskeletal Statistics Unit, The Parker Institute, Bispebjerg and Frederiksberg Hospital, Copenhagen, Denmark

${ }^{3}$ Department of Rheumatology, Odense University Hospital, Odense, Denmark

${ }^{4}$ Department of Neuroscience, Uppsala University, Uppsala, Sweden

${ }^{5}$ Rheumatology and Metabolic Bone Diseases Department, Hospital de Santa Maria, Lisbon, Portugal

${ }^{6}$ Rheumatology Research Unit, Faculty of Medicine, Lisbon Academic Medical Centre, Instituto de Medicina Molecular, University of Lisbon, Lisbon, Portugal

${ }^{7}$ Department of Rheumatology, Virginia Mason Medical Center, Seattle, Washington, USA

${ }^{8}$ Norwegian National Unit for Rehabilitation for Rheumatic Patients with Special Needs, NBRR, Diakonhjemmet Hospital, Oslo, Norway

${ }^{9}$ Department of Rehabilitation Medicine, Maastricht University, Maastricht, The Netherlands

${ }^{10}$ Research Institute for Primary Care and Health Sciences, Keele University, Keele, UK

${ }^{11}$ Haywood Academic Rheumatology Centre, Haywood Hospital, Stoke-on-Trent, UK

${ }^{12}$ EULAR Patient Research Partner, Cyprus League Against Rheumatism, Nicosia, Cyprus

${ }^{13}$ Department of Rheumatology, CHU, Clermont Ferrand, Clermont Ferrand, France

${ }^{14}$ Department of Anesthesiology, Division of Pain Research, Chronic Pain \& Fatigue

Research Center, University of Michigan Medical School, Ann Harbor, Michigan, USA

${ }^{15}$ EULAR Social Leagues Patients' Representative, Leuven, Belgium

${ }^{16}$ Section for Outcomes Research, Center for Medical Statistics, Informatics, and

Intelligent Systems, Medical University of Vienna, Vienna, Austria

${ }^{17}$ Department of Health Sciences, University of Applied Sciences FH Campus Wien,

Vienna, Austria

${ }^{18}$ Research group Health Psychology, University of Leuven, Leuven, Belgium

${ }^{19}$ Behavioral Medicine Research, Faculty of Psychology and Neuroscience, Maastricht University, Maastricht, The Netherlands

${ }^{20}$ Department Physical Therapy, Occupational Therapy, Rehabilitation and Physical Medicine, Universidad Rey Juan Carlos, Alcorcón, Spain
${ }^{21}$ Department of Public Health and Community Medicine, Primary Health Care Unit, Institute of Medicine, The Sahlgrenska Academy, University of Gothenburg, Gothenburg, Sweden

Acknowledgements The authors thank Susan Oliver and Christina Opava who initiated this project on behalf of the EULAR-HP and ARHP steering group that also included Linda Ehrlich-Jones, Ricardo Ferreira, Rinie Geenen, Kam Nola, Jan Richardson, Karen Smarr and Ben Smith. The authors also thank Annette Ladefoged de Thurah for guarding the project.

Contributors CLO was the research fellow for the project undertaking the systematic literature review. RG was the project convener and drafted the work. RC was the methodologist supervising the systematic literature review. CLO and RC rated the quality of studies. CLO and JA independently assessed the studies. All authors have contributed substantially by participating in the development of the recommendations and revising the manuscript critically for important intellectual content. All authors approved the final version for publication.

Funding This study was supported by the European League Against Rheumatism (EULAR), Grant reference: HPR029.

Competing interests None declared.

Patient consent Not required.

Provenance and peer review Not commissioned; externally peer reviewed.

Data sharing statement The data analysed in the current study are available from the corresponding author on reasonable request.

(c) Article author(s) (or their employer(s) unless otherwise stated in the text of the article) 2018. All rights reserved. No commercial use is permitted unless otherwise expressly granted.

\section{REFERENCES}

1 Bijlsma JW, Berenbaum F, Lafeber FP. Osteoarthritis: an update with relevance for clinical practice. Lancet 2011;377:2115-26.

2 Cross M, Smith E, Hoy D, et al. The global burden of hip and knee osteoarthritis: estimates from the global burden of disease 2010 study. Ann Rheum Dis 2014;73:1323-30.

3 Cross M, Smith E, Hoy D, et al. The global burden of rheumatoid arthritis: estimates from the global burden of disease 2010 study. Ann Rheum Dis 2014;73:1316-22.

4 Minnock P, FitzGerald O, Bresnihan B. Women with established rheumatoid arthritis perceive pain as the predominant impairment of health status. Rheumatology 2003:42:995-1000.

5 Ryan S, Carr A. Applying the bio-psychosocial model to the management of rheumatic disease. In: Dziedzic K, Hammond A, eds. Rheumatology: Evidence-Based practice for physiotherapists and occupational therapists. Edinburgh: Churchill Livingstone: Elsevier, 2010:63-75.

6 Iversen MD. Rehabilitation interventions for pain and disability in osteoarthritis. Am J Nurs 2012:112:S32-7. 
7 Jakab Z, Tsouros AD. Health 2020--achieving health and development in today's Europe. Cent Eur J Public Health 2014;22:133-8.

8 Backman CL. Arthritis and pain. Psychosocial aspects in the management of arthritis pain. Arthritis Res Ther 2006;8:221.

9 Bergman S. Management of musculoskeletal pain. Best Pract Res Clin Rheumatol 2007:21:153-66.

10 Gatchel RJ, Peng YB, Peters ML, et al. The biopsychosocial approach to chronic pain: scientific advances and future directions. Psychol Bull 2007;133:581-624.

11 Mork PJ, Vik KL, Moe B, et al. Sleep problems, exercise and obesity and risk of chronic musculoskeletal pain: the Norwegian HUNT study. Eur J Public Health 2014:24:924-9.

12 Da Silva JAP, Geenen R, Jacobs JWG. Chronic widespread pain and increased mortality: biopsychosocial interconnections. Ann Rheum Dis 2017:annrheumdis-2017-211893.

13 Pickering ME, Chapurlat R, Kocher L, et al. Sleep Disturbances and Osteoarthritis. Pain Pract 2016;16:237-44.

14 Walsh DA, McWilliams DF. Mechanisms, impact and management of pain in rheumatoid arthritis. Nat Rev Rheumatol 2014;10:581-92.

15 Fernandes L, Hagen KB, Bijlsma JW, et al. EULAR recommendations for the nonpharmacological core management of hip and knee osteoarthritis. Ann Rheum Dis 2013;72:1125-35.

16 McAlindon TE, Bannuru RR, Sullivan MC, et al. OARSI guidelines for the non-surgical management of knee osteoarthritis. Osteoarthritis Cartilage 2014;22:363-88.

17 Zhang W, Doherty M, Arden N, et al. EULAR evidence based recommendations for the management of hip osteoarthritis: report of a task force of the EULAR Standing Committee for International Clinical Studies Including Therapeutics (ESCISIT). Ann Rheum Dis 2005;64:669-81.

18 Baillet A, Vaillant M, Guinot M, et al. Efficacy of resistance exercises in rheumatoid arthritis: meta-analysis of randomized controlled trials. Rheumatology 2012:51:519-27.

19 Fransen M, McConnell S, Harmer AR, et al. Exercise for osteoarthritis of the knee. Cochrane Database Syst Rev 2015;1:CD004376.

20 Andrews NE, Strong J, Meredith PJ. Activity pacing, avoidance, endurance, and associations with patient functioning in chronic pain: a systematic review and metaanalysis. Arch Phys Med Rehabil 2012;93:2109-21.

21 Zangi HA, Ndosi M, Adams J, et al. EULAR recommendations for patient education for people with inflammatory arthritis. Ann Rheum Dis 2015;74:954-62.

22 van der Heijde D, Aletaha D, Carmona L, et al. 2014 Update of the EULAR standardised operating procedures for EULAR-endorsed recommendations. Ann Rheum Dis 2015;74:8-13.

23 Ghogomu EA, Maxwell LJ, Buchbinder R, et al. Updated method guidelines for cochrane musculoskeletal group systematic reviews and metaanalyses. I Rheumatol 2014:41:194-205.

24 Combe B, Landewe R, Daien $\mathrm{Cl}$, et al. 2016 update of the EULAR recommendations for the management of early arthritis. Ann Rheum Dis 2017;76:948-59.

25 Gossec L, Smolen JS, Ramiro S, et al. European League Against Rheumatism (EULAR) recommendations for the management of psoriatic arthritis with pharmacological therapies: 2015 update. Ann Rheum Dis 2016:75:499-510.

26 Jordan KM, Arden NK, Doherty M, et al. EULAR Recommendations 2003: an evidence based approach to the management of knee osteoarthritis: report of a Task Force of the Standing Committee for International Clinical Studies Including Therapeutic Trials (ESCISIT). Ann Rheum Dis 2003;62:1145-55.

27 Smolen JS, Landewé R, Bijlsma J, et al. EULAR recommendations for the management of rheumatoid arthritis with synthetic and biological disease-modifying antirheumatic drugs: 2016 update. Ann Rheum Dis 2017:76:960-77.

28 van der Heijde D, Ramiro S, Landewé R, et al. 2016 update of the ASAS-EULAR management recommendations for axial spondyloarthritis. Ann Rheum Dis 2017;76:978-91.

29 Zhang W, Doherty M, Leeb BF, et al. EULAR evidence based recommendations for the management of hand osteoarthritis: report of a Task Force of the EULAR Standing Committee for International Clinical Studies Including Therapeutics (ESCISIT). Ann Rheum Dis 2007:66:377-88.

30 Dworkin RH, Turk DC, Farrar JT, et al. IMMPACT. Core outcome measures for chronic pain clinical trials: IMMPACT recommendations. Pain 2005:113:9-19.

31 Treede RD, Rief W, Barke A, et al. A classification of chronic pain for ICD-11. Pain 2015;156:1-7

32 Guyatt G, Oxman AD, Akl EA, et al. GRADE guidelines: 1. Introduction-GRADE evidence profiles and summary of findings tables. J Clin Epidemio/ 2011:64:383-94.

33 Balshem H, Helfand M, Schünemann HJ, et al. GRADE guidelines: 3. Rating the quality of evidence. J Clin Epidemiol 2011;64:401-6.

34 de Jong Z, Vliet Vlieland TP. Safety of exercise in patients with rheumatoid arthritis. Curr Opin Rheumatol 2005;17:177-82.

35 Regnaux JP, Lefevre-Colau MM, Trinquart L, et al. High-intensity versus low-intensity physical activity or exercise in people with hip or knee osteoarthritis. Cochrane Database Syst Rev 2015:CD010203.

36 Lee YC, Lu B, Edwards RR, et al. The role of sleep problems in central pain processing in rheumatoid arthritis. Arthritis Rheum 2013:65:59-68.
37 Irwin MR, Olmstead R, Carrillo C, et al. Sleep loss exacerbates fatigue, depression, and pain in rheumatoid arthritis. Sleep 2012:35:537-43.

38 Vitiello MV, McCurry SM, Shortreed SM, et al. Short-term improvement in insomnia symptoms predicts long-term improvements in sleep, pain, and fatigue in older adults with comorbid osteoarthritis and insomnia. Pain 2014;155:1547-54.

39 Vitiello MV, Rybarczyk B, Von Korff M, et al. Cognitive behavioral therapy for insomnia improves sleep and decreases pain in older adults with co-morbid insomnia and osteoarthritis. J Clin Sleep Med 2009:5:355-62.

40 Smith MT, Finan PH, Buenaver LF, et al. Cognitive-behavioral therapy for insomnia in knee osteoarthritis: a randomized, double-blind, active placebo-controlled clinical trial. Arthritis Rheumatol 2015;67:1221-33.

41 Irwin MR, Cole JC, Nicassio PM. Comparative meta-analysis of behavioral interventions for insomnia and their efficacy in middle-aged adults and in older adults 55+ years of age. Health Psychol 2006;25:3-14.

42 Ho FY, Chung KF, Yeung WF, et al. Self-help cognitive-behavioral therapy for insomnia: a meta-analysis of randomized controlled trials. Sleep Med Rev 2015;19:17-28.

43 van Straten A, van der Zweerde T, Kleiboer A, et al. Cognitive and behavioral therapies in the treatment of insomnia: A meta-analysis. Sleep Med Rev 2018;38:3-16.

44 Koffel EA, Koffel JB, Gehrman PR. A meta-analysis of group cognitive behavioral therapy for insomnia. Sleep Med Rev 2015;19:6-16.

45 Wu JQ, Appleman ER, Salazar RD, et al. Cognitive Behavioral Therapy for Insomnia Comorbid With Psychiatric and Medical Conditions: A Meta-analysis. JAMA Intern Med 2015;175:1461-72.

46 Agca R, Heslinga SC, Rollefstad S, et al. EULAR recommendations for cardiovascular disease risk management in patients with rheumatoid arthritis and other forms of inflammatory joint disorders: 2015/2016 update. Ann Rheum Dis 2017;76:17-28.

47 Strehl C, Bijlsma JW, de Wit M, et al. Defining conditions where long-term glucocorticoid treatment has an acceptably low level of harm to facilitate implementation of existing recommendations: viewpoints from an EULAR task force. Ann Rheum Dis 2016;75:952-7.

48 Smolen JS, Schöls M, Braun J, et al. Treating axial spondyloarthritis and peripheral spondyloarthritis, especially psoriatic arthritis, to target: 2017 update of recommendations by an international task force. Ann Rheum Dis 2018;77:3-17.

49 Singh JA, Saag KG, Bridges SL, et al. 2015 American College of Rheumatology Guideline for the Treatment of Rheumatoid Arthritis. Arthritis Rheumatol 2016;68:1-26

50 Smolen JS, Breedveld FC, Burmester GR, et al. Treating rheumatoid arthritis to target: 2014 update of the recommendations of an international task force. Ann Rheum Dis 2016:75:3-15.

51 Michelsen B, Fiane R, Diamantopoulos AP, et al. A comparison of disease burden in rheumatoid arthritis, psoriatic arthritis and axial spondyloarthritis. PLoS One 2015;10:e0123582

52 Drăgoi RG, Ndosi M, Sadlonova M, et al. Patient education, disease activity and physical function: can we be more targeted? A cross sectional study among people with rheumatoid arthritis, psoriatic arthritis and hand osteoarthritis. Arthritis Res Ther 2013;15:R156.

53 Institute of Medicine (US) Committee on Quality of Health Care in America. Crossing the quality chasm. Washington (DC): National Academies Press (US), 2001.

54 Ammerlaan JW, van Os-Medendorp H, de Boer-Nijhof N, et al. Preferences and needs of patients with a rheumatic disease regarding the structure and content of online self-management support. Patient Educ Couns 2017;100:501-8.

55 Epstein RM, Fiscella K, Lesser CS, et al. Why the nation needs a policy push on patient-centered health care. Health Aff 2010;29:1489-95.

56 Trappenburg J, Jonkman N, Jaarsma T, et al. Self-management: one size does not fit all. Patient Educ Couns 2013:92:134-7.

57 Joosten EA, DeFuentes-Merillas L, de Weert GH, et al. Systematic review of the effects of shared decision-making on patient satisfaction, treatment adherence and health status. Psychother Psychosom 2008;77:219-26.

58 Andersson ML, Svensson B, Bergman S. Chronic widespread pain in patients with rheumatoid arthritis and the relation between pain and disease activity measures over the first 5 years. J Rheumatol 2013;40:1977-85.

59 Clauw DJ. Diagnosing and treating chronic musculoskeletal pain based on the underlying mechanism(s). Best Pract Res Clin Rheumatol 2015;29:6-19.

60 Bergsten U, Bergman S, Fridlund B, et al. "Striving for a good life" - the management of rheumatoid arthritis as experienced by patients. Open Nurs J 2011;5:95-101.

61 Schildmann J, Grunke M, Kalden JR, et al. Information and participation in decisionmaking about treatment: a qualitative study of the perceptions and preferences of patients with rheumatoid arthritis. J Med Ethics 2008:34:775-9.

62 Breivik H, Borchgrevink PC, Allen SM, et al. Assessment of pain. Br J Anaesth 2008:101:17-24.

63 Brummett CM, Bakshi RR, Goesling J, et al. Preliminary validation of the Michigan Body Map. Pain 2016;157:1205-12.

64 Cruccu G, Sommer C, Anand P, et al. EFNS guidelines on neuropathic pain assessment: revised 2009. Eur J Neurol 2010;17:1010-8.

65 Englbrecht M, Tarner IH, van der Heijde DM, et al. Measuring pain and efficacy of pain treatment in inflammatory arthritis: a systematic literature review. J Rheumatol Supp/ 2012;90:3-10. 
66 Hooten WM, Timming R, Belgrade M, et al. Assessment and management of chronic pain. Bloomington: Institute for Clinical Systems Improvement, 2013.

67 Colebatch AN, Edwards CJ, Østergaard M, et al. EULAR recommendations for the use of imaging of the joints in the clinical management of rheumatoid arthritis. Ann Rheum Dis 2013;72:804-14.

68 Mandl P, Navarro-Compán V, Terslev L, et al. EULAR recommendations for the use of imaging in the diagnosis and management of spondyloarthritis in clinical practice. Ann Rheum Dis 2015;74:1327-39.

69 Nelson AE, Allen KD, Golightly YM, et al. A systematic review of recommendations and guidelines for the management of osteoarthritis: The chronic osteoarthritis management initiative of the U.S. bone and joint initiative. Semin Arthritis Rheum 2014;43:701-12.

70 Sullivan MJL, Bishop SR, Pivik J. The Pain Catastrophizing Scale: Development and validation. Psychol Assess 1995; 7:524-32.

71 Goubert L, Crombez G, Van Damme S, et al. Confirmatory factor analysis of the Tampa Scale for Kinesiophobia: invariant two-factor model across low back pain patients and fibromyalgia patients. Clin J Pain 2004;20:103-10.

72 Nicholas MK. The pain self-efficacy questionnaire: Taking pain into account. Eur J Pain 2007:11:153-63.
73 Irish $\mathrm{LA}$, Kline $\mathrm{CE}$, Gunn $\mathrm{HE}$, et al. The role of sleep hygiene in promoting public health: A review of empirical evidence. Sleep Med Rev 2015;22:23-36.

74 Marks R. Obesity profiles with knee osteoarthritis: correlation with pain, disability, disease progression. Obesity 2007;15:1867-74.

75 Egli M, Koob GF, Edwards S. Alcohol dependence as a chronic pain disorder. Neurosci Biobehav Rev 2012:36:2179-92.

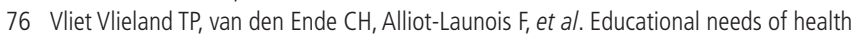
professionals working in rheumatology in Europe. RMD Open 2016:2:e00337.

77 Lillie K, Ryan S, Adams J. The educational needs of nurses and allied healthcare professionals caring for people with arthritis: results from a cross-sectional survey. Musculoskeletal Care 2013:11:93-8.

78 Adams J, Geenen R, Moe RH, et al. Development of the Role and Scope of an Academic Mentorship Network for Health Professionals Working with People with Rheumatological and Musculoskeletal Conditions across Europe. Musculoskeletal Care 2016:14:126-30.

79 Chassany O, Boureau F, Liard F, et al. Effects of training on general practitioners' management of pain in osteoarthritis: a randomized multicenter study. $J$ Rheumatol 2006;33:1827-34.

80 Vlaeyen JW, Linton SJ. Fear-avoidance model of chronic musculoskeletal pain: 12 years on. Pain 2012;153:1144-7. 